\title{
Lifting Dual Connections with the Riemann Extension
}

\section{Stéphane Puechmorel}

ENAC, Université de Toulouse, 31000 Toulouse, France; stephane.puechmorel@enac.fr; Tel.: +33-5-62259503

Received: 7 August 2020; Accepted: 19 November 2020; Published: 21 November 2020

Abstract: Let $(M, g)$ be a Riemannian manifold equipped with a pair of dual connections $\left(\nabla, \nabla^{*}\right)$. Such a structure is known as a statistical manifold since it was defined in the context of information geometry. This paper aims at defining the complete lift of such a structure to the cotangent bundle $T^{*} M$ using the Riemannian extension of the Levi-Civita connection of $M$. In the first section, common tensors are associated with pairs of dual connections, emphasizing the cyclic symmetry property of the so-called skewness tensor. In a second section, the complete lift of this tensor is obtained, allowing the definition of dual connections on $T T^{*} M$ with respect to the Riemannian extension. This work was motivated by the general problem of finding the projective limit of a sequence of a finite-dimensional statistical manifold.

Keywords: information geometry; dual connections; Riemannian extension; cotangent bundle

\section{Introduction}

Information geometry originally dealt with parameter spaces of families of probability densities viewed as differentiable manifolds [1,2]. More specifically, let $E$ be a measure space and let $S=\left\{p_{\theta}, \theta \in\right.$ $M$ \} be a parameterized family of densities on $E$ satisfying:

1. $M$ is a topological manifold (in most cases, it is simply an open subset of $\mathbb{R}^{n}$ ).

2. The topology of $S$ induced by the $L^{1}$ norm is compatible with the topology of $M$.

3. It exists a probability measure $\mu$ on $E$ such that for any $\theta \in M, p_{\theta}<<\mu$.

4. $\quad \theta \mapsto\left(x \in E \rightarrow p_{\theta}(x)\right)$ is smooth uniformly in $x$.

5. $\partial_{\theta} E_{\mu}[\log p(x, \theta)]=E_{\mu}\left[\partial_{\theta} \log p(x, \theta)\right]$.

6. The moments up to order 3 of $x \mapsto \partial_{\theta} \log p(x ; \theta)$ exist and are smooth.

7. The matrix $F$ with entries $F_{i j}(\theta)=E_{p_{\theta}}\left[\partial_{\theta_{i}} \log p(x, \theta) \partial_{\theta_{j}} \log p(x, \theta)\right]$ is positive definite.

The last assumption allows to endow $M$ with the structure of a Riemannian manifold with metric:

$$
g_{\theta}\left(\partial_{\theta_{i}}, \partial_{\theta_{j}}\right)=F_{i j}(\theta)
$$

Parameterized families of the so-called exponential type, the densities of which can be written as:

$$
p(x ; \theta)=\exp (-\langle\theta, T(x)\rangle-\psi(\theta)+h(x))
$$

play a special role in statistics and have a well behaved Riemannian structure. When $T(x)=x$, the family is said to be natural and is defined entirely by $\psi$. In such a case, the Fisher information matrix takes the form:

$$
F_{i j}(\theta)=-E_{p_{\theta}}\left[\frac{\partial^{2}}{\partial \theta_{i} \partial \theta_{j}}\right]
$$

so that the Riemannian metric is Hessian. The structure of such manifolds has been thoroughly studied in [3]. Finally, from considerations arising in statistical estimation, a pair of dual connections 
$\nabla, \nabla^{*}$ with respect to the Fisher metric can be constructed [4]. They possess vanishing torsion and are related by the skewness tensor:

$$
g\left(\nabla_{X} Y, Z\right)-g\left(\nabla_{X}^{*} Y, Z\right)=T(X, Y, Z)
$$

with:

$$
T_{i j k}=E_{p_{\theta}}\left[\partial_{\theta_{i}} \log p(x, \theta) \partial_{\theta_{j}} \log p(x, \theta) \partial_{\theta_{k}} \log p(x, \theta)\right]
$$

As a generalization, a smooth Riemannian manifold $(M, g)$ equipped with a pair $\left(\nabla, \nabla^{*}\right)$ of torsionless dual connections is called a statistical manifold. It can be defined equivalently by $(M, g, T)$ where $T$ is a fully symmetric $(0,3)$-tensor. It turns out [5] that any statistical manifold can be embedded as a statistical model, i.e., one related to a parameterized family of densities.

For a Riemannian manifold $(M, g)$, lifting geometric objects to the tangent bundle TM (resp. cotangent bundle $T^{*} M$ ) is a classical problem [6-8] that relies most of the time on the Whitney sum $T T M=H T M \oplus V T M$ (resp. $T T^{*} M=H T^{*} M \oplus V T^{*} M$ ) with $V T M$ the vertical bundled obtained from the kernel of the canonical projection $d \pi: T T M \rightarrow T M$ (resp. $d \pi: T T^{*} M \rightarrow T^{*} M$ and HTM the horizontal subspace arising from a fixed affine connection $\nabla$. In the tangent bundle, Reference [8] introduces a lift based on horizontal and vertical lifts of vector fields and relies on a quasi-complex structure on $T M$. For $T^{*} M$, the preferred method involves complete lifts [9] and Riemann extensions [10], which are pseudo-Riemannian metrics of neutral signature defined on the cotangent bundle and associated in a canonical way to affine connections with vanishing torsion. The complete lift of the connection is defined to be the Levi-Civita one with respect to its Riemann extension. Complete and vertical lifts of different kinds of tensors are also presented in [6]. Finally, horizontal lifts of connections are presented in [7].

In this paper, the complete lift of dual connections is defined and yields a pair of dual connections, which have vanishing torsion if the original connections do. The strategy adopted is to lift the skewness tensor, here defined in a more general setting as a $(0,3)$-tensor with cyclic symmetry. The procedure described in [6] is adapted to this case, effectively allowing to get a skewness tensor on $T T^{*} M$. The first step is the symmetric lift presented in Definition 6, which yields a (1,2)-tensor on $T T^{*} M$. Applied to the skewness tensor of a pair of dual connections, it yields the mutual torsion of the lifted dual connections. The action of this tensor on vertical and complete lifts of vector fields is given in Proposition 14 and Equation (38). Finally, lifted connections are studied with respect to vertical and complete lifts of forms and vector fields, showing their relationship with mutual curvatures of original dual connections. For a couple of fields $X, Y$ solutions of the equation $\nabla^{*} \nabla=0$, the action of a lifted connection on the complete lifts $X^{c}, Y^{c}$ can be simplified, allowing in a future work a projective limit to be defined.

\section{Statistical Structures}

In information geometry, dual connections are the basic objects defining the so-called statistical manifold structure [4]. The purpose of this section is to recap some properties of statistical structures and to introduce the $(0,3)$-skewness tensor in the context of dual connections with or without torsion. It has the cyclic symmetry property, which is exactly what is needed to lift it as a $(1,2)$-tensor on the cotangent bundle.

In the sequel, $M$ is a smooth $n$-dimensional manifold endowed with a Riemann metric $g$.

Definition 1. Let $\nabla, \nabla^{*}$ be affine connections on $T M$. They are said to be dual if for any triple $X, Y, Z$ of vector fields:

$$
Z(g(X, Y))=g\left(\nabla_{Z} X, Y\right)+g\left(X, \nabla_{Z}^{*} Y\right) .
$$

The torsion of a connection $\nabla$ is the tensor $T$ defined as: $T(X, Y)=\nabla_{X} Y-\nabla_{Y} X-[X, Y]$. If the torsion of $T$ vanishes, so does the torsion of $T^{*}$. 
Proposition 1. Let $\nabla, \nabla^{*}$ be dual connections. Then for any triple $(X, Y, Z)$ :

$$
\left(\nabla_{Z} g\right)(X, Y)=g\left(X, \nabla_{Z}^{*} Y\right)-g\left(X, \nabla_{X} Y\right)
$$

Proof. The covariant derivative of the tensor $g$ is given, for any triple $(X, Y, Z)$ of vector fields by:

$$
\left(\nabla_{Z} g\right)(X, Y)=Z(g(X, Y))-g\left(\nabla_{Z} X, Y\right)-g\left(X, \nabla_{Z} Y\right)
$$

By Definition 1:

$$
Z(g(X, Y))=g\left(\nabla_{Z} X, Y\right)+g\left(X, \nabla_{Z}^{*} Y\right)
$$

So that:

$$
\left(\nabla_{Z} g\right)(X, Y)=g\left(X, \nabla_{Z}^{*} Y\right)-g\left(X, \nabla_{Z} Y\right)
$$

Exchanging $\nabla$ and $\nabla^{*}$, one also has:

$$
\left(\nabla_{Z}^{*} g\right)(X, Y)=g\left(X, \nabla_{Z} Y\right)-g\left(X, \nabla_{Z}^{*} Y\right) .
$$

Thus:

$$
\nabla g=-\nabla^{*} g
$$

Proposition 2. If $\nabla, \nabla^{*}$ are without torsion then the tensor:

$$
(X, Y, Z) \mapsto\left(\nabla_{Z} g\right)(X, Y)
$$

is fully symmetric.

Proof. Symmetry in $X, Y$ is obvious from the symmetry of $g$, while symmetry in $Z$ is obtained from the relations:

$$
\begin{aligned}
& \nabla_{Z} Y=\nabla_{Y} Z-[Y, Z] \\
& \nabla_{Z}^{*} Y=\nabla_{Y}^{*} Z-[Y, Z]
\end{aligned}
$$

Definition 2. Let $\nabla^{1}, \nabla^{2}$ be affine connections on TM. Their mutual torsion is the tensor:

$$
D_{\nabla_{1}, \nabla_{2}}(X, Y)=\nabla_{X}^{1} Y-\nabla_{Y}^{2} X-[X, Y] .
$$

Remark 1. The divergence tensor is defined for dual connections $\nabla, \nabla^{*}$ as $\nabla_{X} Y-\nabla_{X}^{*} Y$. For torsion-less connections, the two notions agree, i.e., $D_{\nabla, \nabla^{*}}=\nabla_{X} Y-\nabla_{X}^{*} Y$.

In the case of dual connections with vanishing torsion, the commutation defect of the divergence is related to the mutual curvature of the connections.

Definition 3. Let $\left(\nabla^{1}, \nabla^{2}\right)$ be a pair of connections. Their mutual curvature is the tensor $(1,3)$-tensor:

$$
R_{\nabla^{1} \nabla^{2}}(X, Y, Z)=\nabla_{X}^{1} \nabla_{Y}^{2} Z-\nabla_{Y}^{1} \nabla_{X}^{2} Z-\nabla_{[X, Y]}^{1} Z
$$

As in the case of the curvature, it is often useful to introduce the $(0,4)$-tensor:

$$
\bar{R}_{\nabla^{1} \nabla^{2}}(X, Y, Z, U)=g\left(R_{\nabla^{1} \nabla^{2}}(X, Y, Z), U\right) .
$$


The curvature and the mutual curvature of dual connections enjoy symmetry properties.

Proposition 3. Let $\left(\nabla, \nabla^{*}\right)$ be a pair of dual connections. Then, for any vector fields $X, Y, Z, U$;

$$
\left\{\begin{array}{l}
\bar{R}(X, Y, Z, U)=\bar{R}^{*}(X, Y, U, Z) \\
\bar{R}_{\nabla^{*} \nabla}(X, Y, Z, U)=\bar{R}_{\nabla \nabla^{*}}(X, Y, U, Z) .
\end{array}\right.
$$

Proof. The proof of the first property is found in, for example, [4]. For the second, the definition of $\bar{R}_{\nabla \nabla^{*}}$ is written as:

$$
\bar{R}_{\nabla^{*} \nabla}(X, Y, Z, U)=g\left(\nabla_{X}^{*} \nabla_{Y} Z, U\right)-g\left(\nabla_{Y}^{*} \nabla_{X} Z, U\right)-g\left(\nabla_{[X, Y]}^{*} Z, U\right) .
$$

Using the duality property:

$$
\begin{aligned}
\bar{R}_{\nabla^{*} \nabla}(X, Y, Z, U)= & X\left(g\left(\nabla_{Y} Z, U\right)\right)-g\left(\nabla_{Y} Z, \nabla_{X} U\right) \\
& -Y\left(g\left(\nabla_{X} Z, U\right)\right)+g\left(\nabla_{X} Z, \nabla_{Y} U\right) \\
& -g\left(\nabla_{[X, Y]}^{*} Z, U\right) .
\end{aligned}
$$

Using duality once again:

$$
\begin{aligned}
\bar{R}_{\nabla^{*} \nabla}(X, Y, Z, U)= & X Y(g(Z, U))-X g\left(Z, \nabla_{Y}^{*} U\right)-Y\left(g\left(Z, \nabla_{X}^{*} U\right)\right)+g\left(Z, \nabla_{Y}^{*} \nabla_{X} U\right) \\
& -Y X(g(Z, U))+Y\left(g\left(Z, \nabla_{X}^{*} U\right)\right) \\
& +X\left(g\left(Z, \nabla_{X}^{*} U\right)\right)-g\left(Z, \nabla_{X}^{*} \nabla_{Y} U\right) \\
& -[X, Y] g(Z, U)+g\left(Z, \nabla_{[X, Y]} U\right)=-\bar{R}_{\nabla \nabla^{*}}^{*}(Y, X, U, Z)=\bar{R}_{\nabla \nabla^{*}}^{*}(X, Y, U, Z) .
\end{aligned}
$$

In the case of dual connections without torsion, the definition of $D(X, Y)$ simplifies to $\nabla_{X} Y-\nabla^{*} X_{Y}$. Letting $D_{X}: Y \rightarrow D(X, Y)$, the next proposition relates the commutation defect to the curvatures.

Proposition 4. For any vector fields $X, Y, Z$ :

$$
D_{X} D_{Y} Z-D_{Y} D_{X} Z=R(X, Y, Z)+R^{*}(X, Y, Z)-R_{\nabla \nabla^{*}}(X, Y, Z)-R_{\nabla^{*} \nabla}(X, Y, Z) .
$$

Proof. By simple computation:

$$
\begin{aligned}
D_{X} D_{Y} Z-D_{Y} D_{X} Z= & \left(\nabla_{X}-\nabla_{X}^{*}\right)\left(\nabla_{Y} Z-\nabla_{Y}^{*} Z\right)-\left(\nabla_{Y}-\nabla_{Y}^{*}\right)\left(\nabla_{X} Z-\nabla_{X}^{*} Z\right) \\
& =\nabla_{X} \nabla_{Y} Z-\nabla_{X} \nabla^{*} Y Z-\nabla_{X}^{*} \nabla_{Y} Z+\nabla_{X}^{*} \nabla_{Y}^{*} Z \\
& -\nabla_{Y} \nabla_{X} Z+\nabla_{Y} \nabla_{X}^{*} Z+\nabla_{Y}^{*} \nabla_{X} Z-\nabla_{Y}^{*} \nabla_{X}^{*} Z
\end{aligned}
$$

and the claims follows by identification of the terms.

Proposition 5. Let $\nabla, \nabla^{*}$ be dual affine connections on $T M$. Then, for any triple $X, Y, Z$ of vector fields:

$$
g\left(\nabla_{X} Y, Z\right)=g\left(\nabla_{X}^{l c} Y, Z\right)+\frac{1}{2}\left[g\left(D_{\nabla, \nabla^{*}}(Z, X), Y\right)-g\left(D_{\nabla, \nabla^{*}}(Y, Z), X\right)+g\left(D_{\nabla, \nabla^{*}}(X, Y), Z\right)\right]
$$

where $\nabla^{l c}$ is the Levi-Civita connection.

Proof. Since the two connections are dual:

$$
X(g(Y, Z))=g\left(\nabla_{X} Y, Z\right)+g\left(Y, \nabla_{X}^{*} Z\right)
$$


Using the definition of $D_{\nabla, \nabla^{*}}$ it becomes:

$$
X(g(Y, Z))=g\left(\nabla_{X} Y, Z\right)+g\left(Y, \nabla_{Z} X\right)-g\left(D_{\nabla, \nabla^{*}}(Z, X), Y\right)-g([Z, X], Y) .
$$

Then, using an alternating sum over the cyclic permutations of $(X, Y, Z)$ and the Koszul formula:

$$
\begin{aligned}
2 g\left(\nabla_{X}^{l c} Y, Z\right)= & X(g(Y, Z))-Z(g(X, Y))+Y(g(Z, X)) \\
& +g(Y,[Z, X]-g(X,[Y, Z])+g(Z,[X, Y])
\end{aligned}
$$

yields the result.

Remark 2. Proposition 5 is the analogue of the Kozsul formula for dual connections. It is a defining property given $D_{\nabla, \nabla^{*}}$.

Notation 1. The (0,3)-tensor:

$$
U_{\nabla_{1}, \nabla_{2}}(X, Y, Z)=g\left(D_{\nabla_{1}, \nabla_{2}}(Z, X), Y\right)-g\left(D_{\nabla_{1}, \nabla_{2}}(Y, Z), X\right)+g\left(D_{\nabla_{1}, \nabla_{2}}(X, Y), Z\right)
$$

is the skewness tensor associated to the connections $\nabla_{1}, \nabla_{2}$. When no confusion is possible in the case of dual connections, the subscripts will be dropped so that $U(X, Y, Z)$ stands for $U_{\nabla, \nabla^{*}}(X, Y, Z)$

Remark 3. The formula of Proposition 5 can be rewritten to give the expression of $\nabla^{*}$ :

$$
g\left(\nabla_{X}^{*} Y, Z\right)=g\left(\nabla_{X}^{l c} Y, Z\right)-\frac{1}{2} U(Y, X, Z) .
$$

Proposition 6. For any triple $(X, Y, Z)$ :

$$
U(X, Y, Z)=U(Y, X, Z)+2 g(T(X, Y), Z)
$$

where $T$ is the torsion of $\nabla$.

Proof. Using the definition:

$$
\nabla_{X} Y=\nabla_{Y} X+[X, Y]+T(X, Y)
$$

and the fact that the Levi-Civita has vanishing torsion:

$$
g\left(\nabla_{X} Y, Z\right)=g\left(\nabla_{X}^{l c} Y, Z\right)+\frac{1}{2} U(X, Y, Z)
$$

Thus:

$$
\begin{aligned}
g\left(\nabla_{Y} X, Z\right) & =g\left(\nabla_{Y}^{l c} X, Z\right)-g(T(X, Y), Z)+\frac{1}{2} U(X, Y, Z) \\
& =g\left(\nabla_{Y}^{l c} X, Z\right)+\frac{1}{2} U(Y, X, Z)
\end{aligned}
$$

and so:

$$
U(X, Y, Z)=U(Y, X, Z)+2 g(T(X, Y), Z)
$$

Proposition 7. The tensor $U$ has the cyclic symmetry propery, that is for any triple $(X, Y, Z)$ of vector fields:

$$
U(X, Y, Z)=U(Z, X, Y)
$$


Proof. Using the symmetry of the Riemann metric, the same derivation as in Proposition 5 but applied to the terms $X(g(Z, Y), Y(g(X, Z), Z(g(Y, X)$ yields:

$$
\begin{aligned}
2 g\left(\nabla_{X}^{l c} Z, Y\right)= & 2 g\left(\nabla_{X} Z, Y\right) \\
& -g(Z, D(Y, X))+g(X, D(Z, Y))-g(Y, D(X, Z)) .
\end{aligned}
$$

By identification it becomes:

$$
U(X, Z, Y)=U(Y, X, Z)
$$

Proposition 8. Let $U$ be a tensor with cyclic symmetry, then the connections defined by:

$$
\begin{aligned}
& g\left(\nabla_{X} Y, Z\right)=g\left(\nabla_{X}^{l c} Y, Z\right)+\frac{1}{2} U(X, Y, Z) \\
& g\left(\nabla_{X}^{*} Y, Z\right)=g\left(\nabla_{X}^{l c} Y, Z\right)-\frac{1}{2} U(Y, X, Z) .
\end{aligned}
$$

are dual

Proof. For any triple $(X, Y, Z)$ of vector fields:

$$
X(g(Y, Z))=g\left(\nabla_{X}^{l c} Y, Z\right)+g\left(Y, \nabla_{X}^{l c} Z\right)
$$

Under the assumption of Equation (10), it becomes:

$$
\begin{aligned}
X(g(Y, Z))= & g\left(\nabla_{X} Y, Z\right)+\frac{1}{2} U(X, Y, Z) \\
& +g\left(\left(Y, \nabla_{X}^{*} Z\right)-\frac{1}{2} U(Z, X, Y)\right.
\end{aligned}
$$

and since $U$ has cyclic symmetry:

$$
X(g(Y, Z))=g\left(\nabla_{X} Y, Z\right)+g\left(\left(Y, \nabla_{X}^{*} Z\right) .\right.
$$

Proposition 9. Let $\nabla_{1}, \nabla_{2}$ be a pair of affine connections. For any triple $(X, Y, Z)$ of vector fields:

$$
g\left(Y, D_{\nabla_{1}, \nabla_{2}}(Z, X)\right)=\frac{1}{2}\left[U_{\nabla_{1}, \nabla_{2}}(X, Y, Z)+U_{\nabla_{1}, \nabla_{2}}(Z, X, Y)\right] .
$$

Proof. Direct computation from the definition of $U$.

Remark 4. Proposition 9 shows that the mutual torsion of a pair of dual connections is uniquely defined by a cyclic symmetric tensor. Conversely, for a pair $\nabla_{1}, \nabla_{2}$ of connections, the cyclic symmetry defect of the tensor $U_{\nabla_{1}, \nabla_{2}}$, namely $A(X, Y, Z)=U_{\nabla_{1}, \nabla_{2}}(X, Y, Z)-U_{\nabla_{1}, \nabla_{2}}(Z, X, Y)$ is the obstruction of being dual. Please note also that the torsion for a pair of dual connections can be seen as the obstruction for the tensor $U$ to be totally symmetric.

Remark 5. A statistical manifold may be defined as a quadruple $(M, g, \nabla, U)$ with $M$ a smooth manifold, $g$ a Riemannian metric, $\nabla$ an affine connection and $U$ a tensor with cyclic symmetry. It slightly more general than the usual definition since $U$ is not required to be totally symmetric, thus allowing connections with torsion. 


\section{Dual Connections Lifts}

Let $U$ be a coordinate neighborhood in $M$ and let $\pi: T^{*} M \rightarrow M$ be the canonical projection. $\phi^{-1}(U)$ is a coordinate neighborhood in $T^{*} M$ with coordinates denoted as $\left(x^{1}, \ldots, x^{n}, p_{1}, \ldots, p_{n}\right)$.

The lift of connections on the cotangent bundle has been studied in [6,7] using the Riemann extension defined in [10]. Another kind of lift is introduced in [11] along with a metric on $T^{*} M$. Let $(M, g)$ be a smooth Riemannian manifold and let $\nabla$ be an affine connection. The kernel of $d \pi: T T^{*} M \rightarrow T^{*} M$ defines an integrable distribution, called the vertical distribution, hereafter denoted by $V T^{*} M$. It is spanned by the vectors:

$$
e_{j+n}=\delta^{j}=\frac{\partial}{\partial p_{j}}, j=1 \ldots n
$$

Complementary to it, there is a horizontal distribution spanned by the vectors:

$$
e_{j}=\partial_{j}+\Gamma_{j i}^{k} p_{k} \delta^{i}, j=1 \ldots n
$$

with:

$$
\partial_{j}=\frac{\partial}{\partial x^{j}} .
$$

These basis vectors are conveniently put into a matrix form, following the convention of [11]:

$$
L=\left(\begin{array}{cc}
I d & 0 \\
\Gamma & I d
\end{array}\right)
$$

where $\Gamma$ is the matrix with entries:

$$
\Gamma_{j i}=\Gamma_{j i}^{k} p_{k}
$$

Definition 4. The Riemannian extension of a torsion-free affine connection $\nabla$ on TM is the symmetric $(0,2)$-tensor with a component matrix:

$$
\nabla^{R}=\left(\begin{array}{cc}
-2 \Gamma & I d \\
I d & 0
\end{array}\right)
$$

where $\Gamma$ is the matrix defined in (16).

Proposition 10. Let $\nabla$ be a torsion-free affine connection on $M$ and let $\left(e_{j}\right)_{1, \ldots, 2 n}$ be its adapted frame in $T^{*} M$. With respect to it, the component matrix of the Riemannian extension is:

$$
\left(\begin{array}{cc}
0 & I d \\
I d & 0
\end{array}\right)
$$

Proof. In the adapted frame, the expression of the component matrix of the Riemannian extension is:

$$
L^{t}\left(\begin{array}{cc}
-2 \Gamma & I d \\
I d & 0
\end{array}\right) L
$$

which is equal to:

$$
\left(\begin{array}{cc}
-2 \Gamma+\Gamma+\Gamma^{t} & I d \\
I d & 0
\end{array}\right)
$$

using the assumption that $\nabla$ is torsion-free, $\Gamma^{t}=\Gamma$ and the claim follows. 
Definition 5. The Levi-Civita connection with respect to the Riemannian extension, denoted by $\nabla^{c}$, is called the complete lift of the connection $\nabla$.

Proposition 11. The Christoffel symbols of the complete lift $\nabla^{c}$ are given by:

$$
{ }^{c} \Gamma_{j i}^{k}=\Gamma_{j i}^{k}{ }^{c} \Gamma_{j i}^{k+n}=p_{l} R_{k i j}^{l},{ }^{c} \Gamma_{j(i+n)}^{k+n}=-\Gamma_{j k}^{i}, i, j, k=1, \ldots, n .
$$

When $\nabla={ }^{l c} \nabla$, the torsion-free assumption is automatically satisfied, so that in an adapted frame the Riemannian extension reduces to the one of Proposition 10.

Proposition 12. Let $\left(\nabla, \nabla^{*}\right)$ be a pair of dual affine connections on $T M$. Then, with respect to the Riemannian extension ${ }^{l c} \nabla^{R}$ of ${ }^{l c} \nabla$, the following relations hold:

$$
\begin{aligned}
L^{t} \nabla^{R} L^{*} & =L^{* t} \nabla^{R} L=\left(\begin{array}{cc}
0 & I d \\
I d & 0
\end{array}\right) \\
L^{t} \nabla^{R} L & =\left(\begin{array}{cc}
\frac{1}{2}\left(\tilde{D}+\tilde{D}^{t}\right) & I d \\
I d & 0
\end{array}\right) \\
L^{* t} \nabla^{R} L^{*} & =\left(\begin{array}{cc}
-\frac{1}{2}\left(\tilde{D}+\tilde{D}^{t}\right) & I d \\
I d & 0
\end{array}\right)
\end{aligned}
$$

where $\tilde{D}$ is the matrix with entries:

$$
\tilde{D}_{j i}=p_{k} D_{j i}^{k} .
$$

and $L\left(\right.$ resp. $\left.L^{*}\right)$ is the component matrix of the adapted frame to $\nabla\left(\right.$ resp. $\left.\nabla^{*}\right)$.

Proof. In the case of dual connections, Equation (12) yields:

$$
g(D(X, Y), Z)=U(X, Y, Z)
$$

and so:

$$
\begin{aligned}
& \nabla={ }^{l c} \nabla+\frac{1}{2} D \\
& \nabla^{*}={ }^{l c} \nabla-\frac{1}{2} D^{t}
\end{aligned}
$$

where $D^{t}(X, Y)=D(Y, X)$. From (20) (resp. (21)), it comes:

$$
\begin{aligned}
& \Gamma={ }^{l c} \Gamma+\frac{1}{2} \tilde{D} \\
& \Gamma^{*}={ }^{l c} \Gamma-\frac{1}{2} \tilde{D}^{t} .
\end{aligned}
$$

We then have:

$$
\nabla^{R} L=\left(\begin{array}{cc}
-{ }^{l c} \Gamma+\frac{\tilde{D}}{2} & I d \\
I d & 0
\end{array}\right)
$$

and:

$$
L^{* t} \nabla^{R} L=\left(\begin{array}{cc}
-\frac{\tilde{D}}{2}+\frac{\tilde{D}}{2} & I d \\
I d & 0
\end{array}\right)=\left(\begin{array}{cc}
0 & I d \\
I d & 0
\end{array}\right) .
$$

The other equations are proved the same way. 
The above relations show that the horizontal subspaces of $\nabla$ and $\nabla^{*}$ are related by the Riemannian extension in a very simple way. Let $X, Y$ be a vector in $T_{x, p} T^{*} M$ with decomposition $X=X_{V}+X_{H}$ (resp. $\left.Y=Y_{V^{*}}+Y_{H^{*}}\right)$ according to the horizontal subspace of $\nabla$ (resp. $\left.\nabla^{*}\right)$, then:

$$
\nabla^{R}(Y, X)=\left\langle Y_{V^{*}}, X_{H}\right\rangle+\left\langle X_{V}, Y_{H^{*}}\right\rangle
$$

with $\langle\cdot, \cdot\rangle$ the Euclidean inner product.

Another interesting fact is that with respect to the adapted frames of $\nabla$ (resp. $\nabla)$, the Riemannian extension becomes a modified Riemannian extension in the sense of [12]. To a given modified Riemannian extension, it is thus possible to associate a pair of dual connections with a given torsion (this last restriction comes from the fact that only the symmetric part of the tensor $D$ enters the expression).

Since duality is related to metric, it is not so obvious how to lift a pair of mutually dual connections in a canonical way since the complete lifts of $\nabla$ and $\nabla^{*}$ involve different Riemannian extensions. The preferred approach will be thus to lift the mutual torsion $D$ to a $(0,3)$-tensor, which can be done by extending the approach of [6], and to exploit the fact that it has a cyclic symmetry property.

In the sequel, the symmetric (resp. anti-symmetric) part with respect to the contravariant indices of the $(1,2)$-tensor $D$ will be denoted by ${ }^{s} D$ (resp. $\left.{ }^{a} D\right)$, i.e.,:

$$
\begin{aligned}
& { }^{s} D_{i j}^{k}=\frac{1}{2}\left(D_{i j}^{k}+D_{j i}^{k}\right) \\
& { }^{a} D_{i j}^{k}=\frac{1}{2}\left(D_{i j}^{k}-D_{j i}^{k}\right) .
\end{aligned}
$$

Proposition 13. The expression:

$$
\sigma=\frac{1}{2} p_{k}^{a} D_{i j}^{k} d x^{i} \wedge d x^{j}
$$

defines a 2 -form on $\mathrm{TT}^{*} M$. Its exterior derivative $d \sigma$ is given by:

$$
d \sigma=\frac{1}{2} p_{l} \frac{\partial^{a} D_{i j}^{l}}{\partial x^{k}} d x^{k} \wedge d x^{i} \wedge d x^{j}+\frac{1}{2}^{a} D_{i j}^{k} d p_{k} \wedge d x^{i} \wedge d x^{j} .
$$

Rearranging the terms, the form $d \sigma$ can be rewritten as:

$$
\begin{aligned}
6 d \sigma= & p_{l}\left(\frac{\partial^{a} D_{i j}^{l}}{\partial x^{k}}+\frac{\partial^{a} D_{k i}^{l}}{\partial x^{j}}+\frac{\partial^{a} D_{j k}^{l}}{\partial x^{i}}\right) d x^{i} \wedge d x^{j} \wedge d x^{k} \\
& +{ }^{a} D_{i j}^{k} d p_{k} \wedge d x^{i} \wedge d x^{j}+{ }^{a} D_{j k}^{i} d x^{k} \wedge d p_{i} \wedge d x^{j}+{ }^{a} D_{k i}^{j} d x^{k} \wedge d x^{i} \wedge d p_{j} .
\end{aligned}
$$

It turns out that the above tensor has cyclic symmetry since it is $(0,3)$ and skew-symmetric. This can be made more explicit by first noticing that the first line on the right hand side obviously has this property. In the second line, considering as an example the first term ${ }^{a} D_{i j}^{k} d p_{k} \wedge d x^{i} \wedge d x^{j}$, a cyclic permutation of the arguments yields ${ }^{a} D_{i j}^{k} d x^{j} \wedge d p^{k} \wedge d x^{i}$. Now, the indices change $j \rightarrow k, k \rightarrow i, i \rightarrow j$ gives ${ }^{a} D_{j k}^{i} d x^{k} \wedge d p_{i} \wedge d x^{j}$, which is exactly the original second term. The remaining terms can be worked the same way.

Considering now the symmetric part of $D$, a similar procedure can applied to obtain a fully symmetric $(0,3)$-tensor. Let us denote by $\odot$ the symmetric tensor product, that is:

$$
x \odot y=(x \otimes y+y \otimes x) / 2 .
$$

From ${ }^{s} D$, a symmetric tensor on $T T^{*} M$ can be defined as:

$$
\theta=\frac{1}{2} p_{k}^{s} D_{i j}^{k} d x^{i} \odot d x^{j}
$$


Following the construction of Proposition 13 and the formula of [13], a fully symmetric lift can be defined.

Definition 6. The symmetric lift of ${ }^{s} D$ is the (0,3)-tensor with components:

$$
\begin{aligned}
& \frac{1}{6}\left(p_{l}\left(\frac{\partial^{s} D_{i j}^{l}}{\partial x^{k}}+\frac{\partial^{s} D_{k i}^{l}}{\partial x^{j}}+\frac{\partial^{s} D_{j k}^{l}}{\partial x^{i}}\right) d x^{i} \odot d x^{j} \odot d x^{k}\right. \\
& \left.+{ }^{s} D_{i j}^{k} d p_{k} \odot d x^{i} \odot d x^{j}+{ }^{s} D_{j k}^{i} d x^{k} \odot d p_{i} \odot d x^{j}+{ }^{s} D_{k i}^{j} d x^{k} \odot d x^{i} \odot d p_{j}\right) .
\end{aligned}
$$

Gathering things together, both the symmetric and the anti-symmetric part of $D$ can be lifted to a cyclic symmetric $(0,3)$-tensor. In the sequel, the notation of [6] is adopted: Latin letters $i, j, \ldots$ refer to $x$ components, overlined letters $\bar{i}, \bar{j}, \ldots$ refers to $p$ components and capital letters can be used for both. As an example, $d x^{\bar{i}}=d p_{i}, \delta^{i}=\partial_{\bar{i}}$.

Definition 7. The cyclic symmetric complete lift of the $(1,2)$-tensor $D$, denoted $U^{c}$, is the $(0,3)$-tensor with components $u_{A B C}^{c} d x^{A} \otimes d x^{B} \otimes d x^{C}$ :

$$
\left\{\begin{array}{l}
u_{i j k}^{c}=p_{l}\left(\frac{\partial D_{i j}^{l}}{\partial x^{k}}+\frac{\partial D_{k i}^{l}}{\partial x^{j}}+\frac{\partial D_{j k}^{l}}{\partial x^{i}}\right) \\
u_{\bar{i} j k}^{c}=D_{j k}^{i} ; u_{i j k}^{c}=D_{k i}^{j} ; u_{i j \bar{k}}^{c}=D_{i j}^{k} \\
u_{i j A}^{\mathcal{c}}=u_{i \bar{i} A \bar{j}}^{c}=u_{A \bar{i} j}^{c}=0 .
\end{array}\right.
$$

Let ${ }^{l c} \nabla^{R}$ be the Riemann extension of the Levi-Civita connection. From $U^{c}$, the complete lift of $D$ can be defined as the $(1,2)$-tensor $D^{c}$ such that for any triple of vector fields:

$$
{ }^{l c} \nabla^{R}\left(X, D^{c}(Y, Z)\right)=U^{c}(X, Y, Z) .
$$

Given the matrix form of the Riemannian extension for the Levi-Civita connection:

$$
{ }^{l c} \nabla^{R}=\left(\begin{array}{cc}
-2^{l c} \Gamma & I d \\
I d & 0
\end{array}\right)
$$

its inverse is readily obtained as:

$$
{ }^{l c} \Delta=\left(\begin{array}{cc}
0 & I d \\
I d & 2 \Gamma
\end{array}\right) .
$$

The components of $D^{c}$ in coordinates can be obtained by composing the matrix $A$, yielding:

$$
\begin{gathered}
D_{A B}^{c C}={ }^{l c} \Delta^{C D} u_{D A B} \\
\left\{\begin{array}{l}
D_{j k}^{c i}=D_{j k}^{i} \\
D_{j k}^{c \bar{i}}=p_{l}\left(\frac{\partial D_{i j}^{l}}{\partial x^{k}}+\frac{\partial D_{k i}^{l}}{\partial x^{j}}+\frac{\partial D_{j k}^{l}}{\partial x^{i}}\right)+2^{l c} \Gamma_{l}^{\bar{i}} D_{j k}^{l} \\
D_{\bar{j} k}^{c \bar{i}}=D_{k i}^{j} \\
D_{j \bar{k}}^{c \bar{i}}=D_{i j}^{k} \\
D_{\bar{j} k}^{c i}=D_{j \bar{k}}^{c i}=D_{\bar{j} k}^{c i}=D_{\bar{j} k}^{c \bar{i}}=0
\end{array}\right.
\end{gathered}
$$

with the notation ${ }^{l c} \Gamma_{l}^{\bar{i}}={ }^{l c} \Gamma_{i l}$. Please note that the above relations are different from the one given in [6] for the complete lift of a skew-symmetric $(1,2)$-tensor since here the Riemann extension is used 
in place of the canonical $(1,1)$-tensor $\epsilon$ and only the cyclic symmetry is assumed. This last fact can be noticed in the third and fourth lines of Equation (28).

The next definitions are recalled for the sake of completeness.

Definition 8. Let $\omega=\omega_{i} d x^{i}$ be a degree 1 differential form. Its vertical lift to $T T^{*} M$ is the vector field:

$$
\omega^{V}=\omega_{i} \delta^{i}
$$

The vector fields admit both a vertical and a complete lift. Only the later will be used here.

Definition 9. Let $X=X^{i} \partial_{i}$ be a vector field on $M$. Its complete lift to $T T^{*} M$ is the vector field:

$$
X^{c}=X^{i} \partial_{i}-p_{l} \frac{\partial X^{l}}{\partial x^{k}} \delta^{k}
$$

Finally $(1,1)$-tensors can be lifted in a quite obvious way:

Definition 10. Let $F$ be a $(1,1)$-tensor field. Its vertical lift to $T T^{*} M$ is the vector field:

$$
F^{V}=p_{l} F_{k}^{l} \delta^{k}
$$

The action of $D^{c}$ on vertical and complete lift can now be obtained.

Proposition 14. Let $X$ be a vector field and $\omega, \theta$ be 1-forms. Then:

$$
\left\{\begin{array}{l}
D^{c}\left(\omega^{V}, \theta^{V}\right)=0 \\
D^{c}\left(\omega^{V}, X^{c}\right)=\left(\omega D_{X}\right)^{V}, D^{c}\left(X^{c}, \omega^{V}\right)=\left(\omega D^{X}\right)^{V}
\end{array}\right.
$$

where $D_{X}\left(\right.$ resp. $\left.D^{X}\right)$ is the $(1,1)$-tensor defined by: $D_{X}(Y)=D(X, Y)\left(\right.$ resp. $D^{X}(Y)=D(Y, X)$ ).

Proof. Let $\omega=\omega_{i} d x^{i}, \theta=\theta_{j} d x^{j}$. Then $D\left(\omega^{v}, \theta^{V}\right)=\omega^{\bar{i}} \theta^{\bar{j}} D_{i j}^{c A}=0$. Let $X$ be vector field and $X^{c}$ its complete lift. By linearity:

$$
D^{c A}\left(\omega^{V}, X^{c}\right)=\omega^{\bar{i}} X^{j} D_{\bar{i} j}^{c A}-p_{l} \frac{\partial X^{l}}{x^{k}} D_{\bar{i} \bar{k}}^{c A}
$$

Since $D_{\bar{i} \hat{k}}^{c A}=0$, the second term in the right hand side vanishes. For the fist one, only $D_{\bar{i} j}^{c \bar{k}}=D_{j k}^{i}$ is non-zero, so that:

$$
D^{c}\left(\omega^{V}, X^{c}\right)=\omega_{i} X^{j} D_{j k}^{i} \delta^{k}
$$

The tensor $D_{X}$ has expression $D_{X}(Y)=D_{i j}^{k} X^{i} Y^{j} \partial_{k}$, so that $\omega D_{X}$ is the form $\omega D_{X}=\omega_{k} X^{i} D_{i j}^{k} d x^{j}$, of which the vertical lift is $\omega_{k} X^{i} D_{i j}^{k} z^{k}$.

Please note while the expression obtained is similar to the one of [6], the sign is opposite.

The case of the action on two complete lifts is a little bit more complicated. First of all, given two vector fields $X=X^{i} \partial_{i}, Y=Y^{j} \partial_{j}$, a simple computation yields:

$$
\begin{aligned}
D^{c}\left(X^{c}, Y^{c}\right) & =X^{i} Y^{j} D_{i j}^{k} \partial_{k}+X^{i} Y^{j} p_{l}\left(\frac{\partial D_{i j}^{l}}{\partial x^{k}}+\frac{\partial D_{k i}^{l}}{\partial x^{j}}+\frac{\partial D_{j k}^{l}}{\partial x^{i}}\right) \delta^{k} \\
& +2 p_{l}^{l c} \Gamma_{k l} D_{i j}^{l} X^{i} Y^{j} \delta^{k}-X^{i} p_{l} \frac{\partial Y^{l}}{\partial x^{j}} D_{k i}^{j} \delta^{k}-Y^{j} p_{l} \frac{\partial x^{l}}{\partial x^{i}} D_{j k}^{i} \delta^{k} .
\end{aligned}
$$


After rewriting, Equation (30) becomes:

$$
\begin{aligned}
D^{c}\left(X^{c}, Y^{c}\right) & =X^{i} Y^{j} D_{i j}^{k} \partial_{k}+X^{i} p_{l}\left(Y^{j} \frac{\partial D_{k i}^{l}}{\partial x^{j}}-\frac{\partial Y^{l}}{\partial x^{j}} D_{k i}^{j}\right) \delta^{k} \\
& +Y^{j} p_{l}\left(X^{i} \frac{\partial D_{j k}^{l}}{\partial x^{i}}-\frac{\partial X^{l}}{\partial x^{i}} D_{j k}^{i}\right) \delta^{k}+X^{i} Y^{j} p_{l} \frac{\partial D_{i j}^{l}}{\partial x^{k}} \delta^{k}+2 p_{l}^{l c} \Gamma_{k l} D_{i j}^{l} X^{i} Y^{j} \delta^{k} .
\end{aligned}
$$

Let us consider, for $X, Y$ fixed vector fields, the $(1,1)$-tensor ${ }^{l c} \nabla D(X, Y)$ :

$$
Z \mapsto{ }^{l c} \nabla_{Z}(D(X, Y))=Z^{k} \frac{\partial D_{i j}^{l} X^{i} Y^{j}}{\partial x_{k}}+{ }^{l c} \Gamma_{k m}^{l} D_{i j}^{m} X^{i} Y^{j} Z^{k} .
$$

Its vertical lift is then:

$$
\left({ }^{l c} \nabla D(X, Y)\right)^{V}=p_{l} \frac{\partial D_{i j}^{l} X^{i} Y^{j}}{\partial x^{k}} \delta^{k}+p_{l}^{l c} \Gamma_{k m}^{l} D_{i j}^{m} X^{i} Y^{j} \delta^{k} .
$$

On the other hand, the complete lift of the vector field $D(X, Y)$ is:

$$
(D(X, Y))^{C}=D_{i j}^{k} X^{i} Y^{j} \partial_{k}-p_{l} \frac{\partial D_{i j}^{l} X_{i} Y_{j}}{\partial x^{k}} \delta^{k}
$$

Combining Equations (32) and (33) yields:

$$
2 p_{l}^{l c} \Gamma_{k l} D_{i j}^{l} X^{i} Y^{j} \delta^{k}+X^{i} Y^{j} D_{i j}^{k} \partial_{k}=2\left({ }^{l c} \nabla D(X, Y)\right)^{V}+(D(X, Y))^{C}-p_{l} \frac{\partial D_{i j}^{l} X_{i} Y_{j}}{\partial x^{k}} \delta^{k} .
$$

Putting the expression in Equation (31) yields:

$$
\begin{aligned}
D^{c}\left(X^{c}, Y^{c}\right) & =2\left({ }^{l c} \nabla D(X, Y)\right)^{V}+(D(X, Y))^{C}+X^{i} p_{l}\left(Y^{j} \frac{\partial D_{k i}^{l}}{\partial x^{j}}-\frac{\partial Y^{l}}{\partial x^{j}} D_{k i}^{j}\right) \delta^{k} \\
& +Y^{j} p_{l}\left(X^{i} \frac{\partial D_{j k}^{l}}{\partial x^{i}}-\frac{\partial X^{i}}{\partial x^{i}} D_{j k}^{i}\right) \delta^{k}-p_{l} D_{i j}^{l} \frac{\partial X^{l}}{\partial x^{k}} Y^{j} \delta^{k}-p_{l} D_{i j}^{l} X^{i} \frac{\partial Y_{j}}{\partial x^{k}} \delta^{k} .
\end{aligned}
$$

Let $K$ be a $(1,1)$-tensor $K$. Its Lie derivative can be written [14] (p. 32, Proposition 35):

$$
\mathcal{L}_{X} K(Y)=[X, K(Y)]-K([X, Y]) .
$$

It thus becomes:

$$
\mathcal{L}_{Y} D_{X}(Z)=\left[Y, D_{X}(Z)\right]-D_{X}([Y, Z]),
$$

which can be written in coordinates:

$$
\begin{aligned}
\mathcal{L}_{Y} D_{X}(Z)^{l} & =Y^{j} \frac{\partial D_{i k}^{l} X^{i} Z^{k}}{\partial x^{j}}-D_{i k}^{j} X^{i} \frac{\partial Y^{l}}{\partial x^{j}} Z^{k}-Y^{j} \frac{\partial Z^{k}}{\partial x^{j}} X^{i} D_{i k}^{l}+Z^{j} \frac{\partial Y^{k}}{\partial x^{j}} X^{i} D_{i k}^{l} \\
& =X^{i}\left(Y^{j} \frac{\partial D_{i k}^{l}}{\partial x_{j}}-D_{i k}^{j} \frac{\partial Y^{l}}{\partial x^{j}}\right) Z^{k}+\frac{\partial Y^{k}}{\partial x^{j}} X^{i} D_{i k}^{l} Z^{j} .
\end{aligned}
$$


Plugging it into Equation (35) finally gives the reduced expression:

$$
\begin{aligned}
D^{c}\left(X^{c}, Y^{c}\right) & =2\left(\left({ }^{l c} \nabla D\right)(X, Y)\right)^{V}+(D(X, Y))^{C} \\
& +\left(\mathcal{L}_{Y} D^{X}+\mathcal{L}_{X} D_{Y}\right)^{V} \\
& +2(A(X, Y)+B(X, Y))^{V}
\end{aligned}
$$

where $A(X, Y), B(X, Y)$ are the tensors defined in coordinates by:

$$
\begin{aligned}
& A(X, Y)(Z)^{l}=D_{i j}^{l l c} \Gamma_{m k}^{i} Z^{k} X^{m} Y^{j} \\
& B(X, Y)(Z)^{l}=D_{i j}^{l l c} \Gamma_{m k}^{j} Z^{k} Y^{m} X^{i}
\end{aligned}
$$

The Equation (38) completely defines the tensor $D^{c}$.

From the complete lift $D^{c}$, dual connections with respect to the Riemannian extension can be obtained:

$$
\left\{\begin{array}{l}
\tilde{\nabla}=\nabla^{c}+\frac{1}{2} D^{c} \\
\tilde{\nabla}^{*}=\nabla^{c}-\frac{1}{2} D^{c t} .
\end{array}\right.
$$

The pair $\left(\tilde{\nabla}, \tilde{\nabla}^{*}\right)$ defines the complete lift of the original statistical structure to the pseudo-Riemannian manifold $\left(T^{*} M, \nabla^{R}\right)$. When $\nabla$ is without torsion, then $D$ is symmetric. Using Equation (38) and the fact that in such a case $D_{X}=D^{X}$ show that $D^{c}$ is itself symmetric, proving that $\tilde{\nabla}$ has vanishing torsion.

\section{Relation with Curvature}

From [6], the complete lift $^{l c} \nabla^{c}$ of the Levi-Civita connection with respect to the Riemann extension has components in coordinates:

$$
\left\{\begin{array}{l}
{ }^{l c} \tilde{\Gamma}_{j k}^{i}={ }^{l c} \Gamma_{j k}^{i} \\
{ }^{l c} \tilde{\Gamma}_{j k}^{\bar{i}}=p_{l}\left(\frac{\partial^{l c} \Gamma_{j k}^{l}}{\partial x_{i}}-\frac{\partial^{l c} \Gamma_{k i}^{l}}{\partial x_{j}}-\frac{\partial^{l c} \Gamma_{i j}^{l}}{\partial x_{k}}\right)+2{ }^{l c} \Gamma_{l}^{\bar{i} l c} \Gamma_{j k}^{l} \\
{ }^{l c} \tilde{\Gamma}_{j \bar{k}}^{\bar{i}}=-{ }^{l c} \Gamma_{k i}^{j} \\
{ }^{l c} \tilde{\Gamma}_{\bar{j} k}^{i}=-{ }^{l c} \Gamma_{i j}^{k} \\
{ }^{l c} \tilde{\Gamma}_{\overline{j k}}^{i}={ }^{l c} \tilde{\Gamma}_{j k}^{i}={ }^{l c} \tilde{\Gamma}_{j k}^{i}={ }^{l c} \tilde{\Gamma}_{\overline{j k}}^{\bar{i}}=0
\end{array}\right.
$$

Using the expression (40), and assuming that the connections $\nabla, \nabla^{*}$ are without torsion, the components $\tilde{\Gamma}$ of the lift $\tilde{\nabla}$ in coordinates are:

$$
\left\{\begin{array}{l}
\tilde{\Gamma}_{j k}^{i}=\Gamma_{j k}^{i} \\
\tilde{\Gamma}_{j k}^{i}=p_{l}\left(\frac{\partial \Gamma_{j k}^{l}}{\partial x_{i}}-\frac{\partial \Gamma_{k i}^{* l}}{\partial x_{j}}-\frac{\partial \Gamma_{i j}^{* l}}{\partial x_{k}}\right)+\Gamma_{l}^{\bar{i}} \Gamma_{j k}^{l}+\Gamma_{l}^{* \bar{i}} \Gamma_{j k}^{l} \\
\tilde{\Gamma}_{j \bar{k}}^{\bar{i}}=-\Gamma_{k i}^{* j} \\
\tilde{\Gamma}_{\bar{j} k}^{\bar{i}}=-\Gamma_{i j}^{* k} \\
\tilde{\Gamma}_{j k}^{i}=\tilde{\Gamma}_{j \bar{k}}^{i}=\tilde{\Gamma}_{j k}^{i}=\tilde{\Gamma}_{j k}^{\bar{i}}=0
\end{array}\right.
$$

with $\Gamma$ (resp. $\Gamma^{*}$ ) the components of $\nabla, \nabla^{*}$. The components of $\tilde{\nabla}^{*}$ are obtained by exchanging $\Gamma$ and $\Gamma^{*}$ in the above expression. The effect of $\tilde{\Gamma}$ on vertical and complete lifts of vector fields and forms can be computed as: 


$$
\left\{\begin{array}{l}
\tilde{\nabla}_{\theta^{v}} \omega^{v}=0 \\
\tilde{\nabla}_{X^{c}} \omega^{v}=\left(\nabla_{X}^{*} \omega\right)^{v} \\
\tilde{\nabla}_{\theta^{v}} Y^{c}=-(\theta(\nabla Y))^{v} \\
\tilde{\nabla}_{X^{c}} Y^{c}=\left(\nabla_{X}^{Y}\right)^{c}+W_{X, Y}^{v}
\end{array}\right.
$$

where $W_{X Y}$ is the tensor:

$$
Z \mapsto \nabla_{\nabla_{Z}^{*} Y} X+\nabla_{\nabla_{Z}^{*} X} Y-R_{\nabla \nabla^{*}}(X, Z, Y)-R_{\nabla \nabla^{*}}(Y, Z, X)
$$

Let $(X, Y, Z)$ be vector fields and define $\nabla^{*} \nabla$ as:

$$
\left(\nabla_{Z}^{*} \nabla\right)_{X} Y=\nabla_{\nabla_{Z}^{*} X}-\nabla_{X} \nabla_{Z}^{*} Y
$$

A field $Y$ solution of $\nabla^{*} \nabla Y=0$ is such that for any couple $(Z, X)$ :

$$
\nabla_{\nabla_{Z}^{*} X} Y=\nabla_{X} \nabla_{Z}^{*} Y
$$

The set of solutions of the above equation will be denoted as $J_{\nabla^{*} \nabla}$, following the convention of [15]. When both $X, Y$ are in $J_{\nabla^{*} \nabla}$, the tensor $W_{X, Y}$ simplifies to:

$$
W_{X, Y} Z=\nabla_{Z}\left(\nabla_{X}^{*} Y+\nabla_{Y}^{*} X\right)
$$

It turns out that [6]:

$$
\left(\nabla_{X}^{*} Y+\nabla_{Y}^{*} X\right)^{v}=-\nabla^{* R}\left(X^{c}, Y^{c}\right)
$$

where $\nabla^{* R}$ is the Riemann extension of $\nabla^{*}$. The solutions of the equation $\nabla^{*} \nabla=0$ can thus be transposed to the cotangent bundle using Riemann extensions.

Funding: This research received no external funding.

Conflicts of Interest: The author declares no conflict of interest.

\section{References}

1. Amari, S.; Nagaoka, H. Methods of Information Geometry; Translations of Mathematical Monographs; American Mathematical Society: Providence, RI, USA, 2007.

2. Amari, S. Information Geometry and Its Applications; Applied Mathematical Sciences; Springer: Tokyo, Japan, 2016.

3. Shima, H. The Geometry of Hessian Structures; World Scientific: Singapore, 2007.

4. Amari, S.; Barndorff-Nielsen, O.; Kass, R.; Lauritzen, S.; Rao, C. Differential Geometry in Statistical Inference; American Oriental Series; Institute of Mathematical Statistics: Beachwood, OH, USA, 1987.

5. Le, H. Statistical manifolds are statistical models. J. Geom. 2006, 84, 83-93. [CrossRef]

6. Yano, K.; Patterson, E.M. Vertical and complete lifts from a manifold to its cotangent bundle. J. Math. Soc. Jpn. 1967, 19, 91-113. [CrossRef]

7. Yano, K.; Patterson, E.M. Horizontal lifts from a manifold to its cotangent bundle. J. Math. Soc. Jpn. 1967, 19, 185-198. [CrossRef]

8. Dombrowski, P. On the Geometry of the Tangent Bundle. J. Reine Angew. Math. 1962, 1962, $73-88$. [CrossRef]

9. Satô, I. Complete lifts from a manifold to its cotangent bundle. Kodai Math. Sem. Rep. 1968, 20, 458-468. [CrossRef]

10. Patterson, E.M.; Walker, A. Riemann extensions. Q. J. Math. 1952, 3, 19-28. Available online: https://academic.oup.com/qjmath/article-pdf/3/1/19/7295501/3-1-19.pdf (accessed on 12 May 2020). [CrossRef]

11. Mok, K.P. Metrics and connections on the cotangent bundle. Kodai Math. Sem. Rep. 1977, 28, $226-238$. [CrossRef] 
12. Calviño-Louzao, E.; Garcia-Rio, E.; Gilkey, P.; Vázquez-Lorenzo, R. The geometry of modified Riemannian extensions. Proc. R. Soc. Math. Phys. Eng. Sci. 2009, 465. [CrossRef]

13. Olver, P.J. Symmetry groups and group invariant solutions of partial differential equations. J. Differ. Geom. 1979, 14, 497-542. [CrossRef]

14. Kobayashi, S.; Nomizu, K. Foundations of Differential Geometry; A Wiley Publication in Applied Statistics; Wiley: Hoboken, NJ, USA, 1996; Volume 1.

15. Nguiffo Boyom, M. Foliations-Webs-Hessian Geometry-Information Geometry-Entropy and Cohomology. Entropy 2016, 18, 433. [CrossRef]

Publisher's Note: MDPI stays neutral with regard to jurisdictional claims in published maps and institutional affiliations.

(C) 2020 by the authors. Licensee MDPI, Basel, Switzerland. This article is an open access article distributed under the terms and conditions of the Creative Commons Attribution (CC BY) license (http://creativecommons.org/licenses/by/4.0/). 